\title{
Predication of Gas Emission Based on Wavelet Multi-resolution Analysis
}

\author{
Baoming Qiao \\ Department of Applied Mathematics, Xi'an University \\ of Science and Technology \\ XUST \\ Xi'an China \\ e-mail: qiaobm@xust.edu.cn
}

\author{
Qiao Kang \\ Department of Applied Mathematics, Xi'an University \\ of Science and Technology \\ XUST \\ Xi'an China \\ e-mail: kangqiao_2013@126.com
}

\begin{abstract}
Gas emission is basically non-stationary time series, Based on this view, wavelet multi-resolution analysis was applied to the predication of gas emission. Firstly, the gas emission data was decomposed by wavelet multi-resolution analysis. Secondly, the single branch reconstruction of each layer was predicted by establishing AR forecasting model. Synthesized all of the results from every layers, the forecasting result was obtained. The simulation showed that predication for mine gas emission with the method of this paper has much higher accuracy than AR model forecasting model.
\end{abstract}

Keywords-wavelet multi-resolution analysis; gas emission; time series; AR model; Mallat algorithm; Predication; decomposition; reconstruction

\section{INTRODUCTION}

For poor natural condition and complex geological structure, our China's coal mine accidents often occur, It is provided that mine gas emission is an important indicator of coal mine safety, an indispensable part of gas prevention, and is directly related to safe production of coal and the decision making. At present, forecasting method widely used are Statistic method, different-source method, grey theory predication method and neural network forecasting method. Based on system theory, the series of gas emission dynamic data, according to its data order and its size, inevitably contains traces and large amount of feature information of the dynamic evolution ${ }^{[1]-[2]}$. The common methods of time series predication are autoregressive model AR, moving aver-age model MA and mixed model ARMA, and its premise condition is that the time series is smooth or stationary. But gas emission data are often non-stationary, so direct use of those model lead to the prediction error larger. Aiming at this point, the paper introduced multiresolution analysis into the prediction of time series, because some nonstationary time series can be decomposed into several approximate stationary time series by wavelet multi-resolution analysis.

\section{WAVELET MULTI-RESOLUTION ANALYSIS}

In 1988, S.Mallat proposed the concept of multiresolution analysis (MRA) [3], illustrated the multiresolution characteristics of wavelet from the perspective of function space. Multi-resolution analysis which is also known as multi-scale analysis studied the multi-scale representation of the function or signal from the point of the space. In addition, it also provided a unified framework for constructing wavelet, as well as a fast algorithm of signal decomposition and reconstruction, Mallat algorithm.

Multi-resolution analysis of time series is further decomposing in the low frequency portion at different scales, while the high frequency doesn't deal with, as it shown in Figure 1.

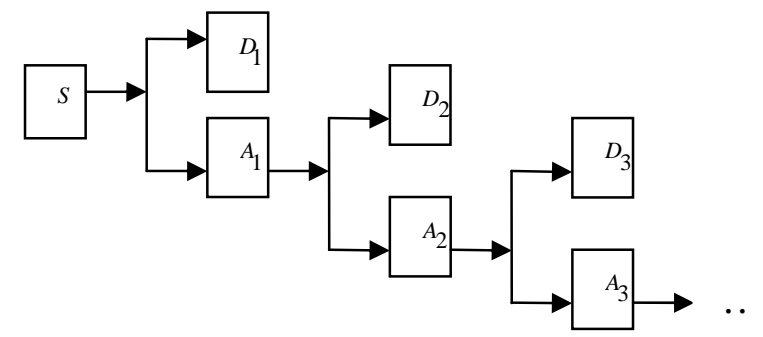

Figure1. Multi-resolution analysis of wavelet decomposition tree graph

Where $A$ represents the decomposition of the low frequency part, and $D$ represents the high frequency part., $j$ in $A_{j}$ and $D_{j}(j=1 \cdots 3)$ is the decomposition layers.

Mallat algorithm ${ }^{[4]-[5]}$ occupies an important position in the multi-resolution analysis. It is the fast algorithm about the construction and transform of orthogonal wavelet. Set the scaling function is $\varphi(t)$, and its corresponding wavelet function is $\psi(t)$, the two-scale equation is

$$
\left\{\begin{array}{l}
\phi_{m, k}(t)=\sum_{l} h_{l-2 k} \phi_{m+1, l}(t) \\
\psi_{m, k}(t)=\sum_{l} g_{l-2 k} \phi_{m+1, l}(t)
\end{array}\right.
$$

Mallat algorithm include decomposition and decomposition algorithm, The decomposition algorithm is shown as following.

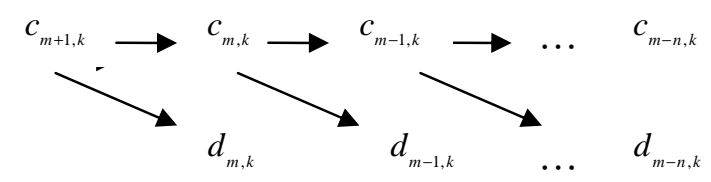

Figure2. Schematic diagram of decomposition algorithm

Mallat reconstruction algorithm is actually the inverse operation of the decomposition algorithm as figure3 showed. 


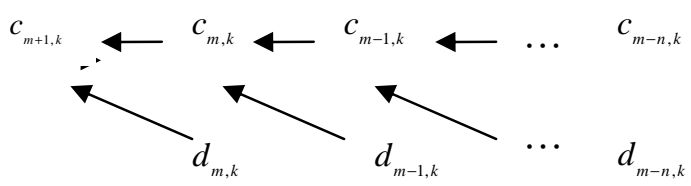

Figure3. Schematic diagram of reconstruction algorithm

\section{AR PREDICTING MODEL}

Set a time series is $\left\{x_{t}\right\}, t=1,2, \cdots, N$, and $\operatorname{AR}(n)$ model $^{[6]}$ is

$$
x_{t}=\varphi_{1} x_{t-1}+\varphi_{2} x_{t-2}+\cdots+\varphi_{n} x_{t-n}+a_{t}, a_{t} \sim \operatorname{NID}\left(0, \sigma_{a}^{2}\right)
$$

In the model above, the unknown parameters is $\varphi_{1}, \varphi_{2}, \cdots \varphi_{n}, \sigma_{a}^{2}$, and the relations is as following

$$
\begin{gathered}
a_{t}=x_{t}-\varphi_{1} x_{t-1}-\varphi_{2} x_{t-2}-\cdots-\varphi_{n} x_{t-n} \\
\sigma_{a}^{2}=\frac{1}{N-n} \sum_{t=n+1}^{N}\left(x_{t}-\sum_{i=1}^{n} \varphi_{i} x_{t-i}\right)^{2}
\end{gathered}
$$

The premise of establishing model is parameter estimate and model test. There are many methods of model test, the paper choose Akaike information criterion, whose basic idea is with model order increasing, the accuracy of model approximation to the real system improves, and $\sigma_{a}^{2}$ decreases. However, the rise of model order means that the number of model parameters increase and leads to the error increases. Consolidated these two aspects, there should be a more appropriate order, which corresponds to model is the suitable one. Akaike information criterion includes FPE criterion, AIC criterion, BIC criterion, $\Phi$ IC criterion, $\Phi$ IC criterion, $\mathrm{BI} \mathrm{C}_{1}$ criterion, CAT criterion. However, these guidelines are only tested from one aspect to test model, and is not comprehensive enough. Guoqiang Chen etc ${ }^{[7]}$ had

proposed a test method that combined three commonly used criterions to determine the model order, which is through drawing FPE, AIC, BIC function with respect to the order $n$, seek the common minimum value which is the model order. It is intuitive and simple to determine the model order.

The paper firstly determined the scope of the order by experience criterion, then used the method above within the scope to determine the model order.

$\operatorname{AR}(n)$ model $x_{t}=\varphi_{1} x_{t-1}+\varphi_{2} x_{t-2}+\cdots+\varphi_{n} x_{t-n}+a_{t}$, its best predictor formula is

$$
\hat{x}_{t}(l)=\left\{\begin{array}{lr}
\sum_{i=1}^{n} \varphi_{i} X_{t+l-i} & (l=1) \\
\sum_{i=1}^{l-1} \varphi_{i} \hat{x}_{t}(l-i)+\sum_{i=1}^{n} \varphi_{i} x_{t+l-i} & (1<l \leq n) \\
\sum_{i=1}^{n} \varphi_{i} \hat{x}_{t}(l-i) & (l>n)
\end{array}\right.
$$

Where $l$ referring to the number of steps, $\hat{x}_{t}(l)$ is the best predicted value of forward $l$ steps in $t$.

\section{PREDICTING METHOD BASED ON MULTI-RESOLUTION ANALYSIS $^{[8]-[10]}$}

Set $S=\left\{x_{1}, x_{2}, \cdots, x_{n}\right\}$ is a non-stationary time series, decomposes $S$ with wavelet, that is only further decomposes the low frequency part of the time series, then single branch reconstructs each layer, the following equation can be achieved.

$$
S=D_{1}+D_{2}+\cdots+D_{i}+A_{i}
$$

Here $i$ is the number of layers, $D_{i}$ is the result of single branch reconstruction of high frequency part, $A_{i}$ is the result of single branch reconstruction of low frequency part. $D_{1}=\left\{d_{1,1}, d_{1,2}, \cdots, d_{1, n}\right\}, D_{2}=\left\{d_{2,1}, d_{2,2}, \cdots, d_{2, n}\right\}, \cdots$, $D_{i}=\left\{d_{i, 1}, d_{i, 2}, \cdots, d_{i, n}\right\}, A_{i}=\left\{a_{i, 1}, a_{i, 2}, \cdots a_{i, n}\right\}$, so the time series can be expressed as

$$
S(t)=d_{1, t}+d_{2, t}+d_{3, t}+\cdots+d_{i, t}+a_{i, t}
$$

Now $\{S(t)\}$ is given, the value after $l$ steps is

$$
\hat{S}(n+l)=\hat{d}_{1, n+1}+\hat{d}_{2, n+1}+\hat{d}_{3, n+1}+\cdots+\hat{d}_{i, n+1}+\hat{a}_{i, n+1}
$$

Here $\hat{d}_{1, n+1}, \hat{d}_{2, n+1}, \hat{d}_{3, n+1}, \cdots, \hat{d}_{i, n+1}, \hat{a}_{i, n+1}$ can be solved through the AR prediction model, solving steps are as follows.

Step1: Determine the model parameters and order for $D_{i}(i=1,2, \cdots, i)$ and $A_{i}$, establish AR model respectively;

Step2: Predict $\hat{d}_{1, n+1}, \hat{d}_{2, n+1}, \hat{d}_{3, n+1}, \cdots, \hat{d}_{i, n+1}$ and $\hat{a}_{i, n+1}$ with the model established in step 1 , then substituted them into the formula (3.3), the predicted value of the time series can be achieved.

\section{THE SIMULATION EXPERIMENT OF GAS EMISSION PREDICTING}

The gas emission data were collected daily from February 13 to December 31 in 2006 in a cool mine. The simulation is divided into two parts: the first part is predicting with AR model, the second part is predicting based on multi-resolution analysis. All the forecasting curves is compared to the original data. The figure below is the original data of gas emission.

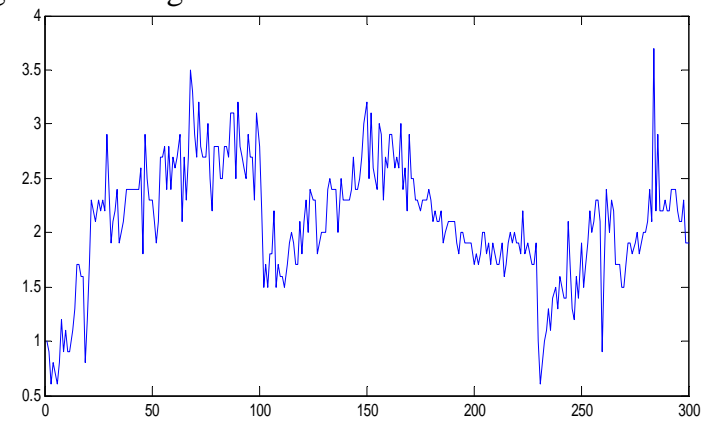

Figure.4 Original data diagram 
It can be seen from Figure. 4 that the fluctuation of gas emission with the changing of time, and it is a nonstationary time series.

\section{A. Prediction of AR model.}

Establish AR model, Fistly, determine the order of the model as III mentioned, the Figure is as following.

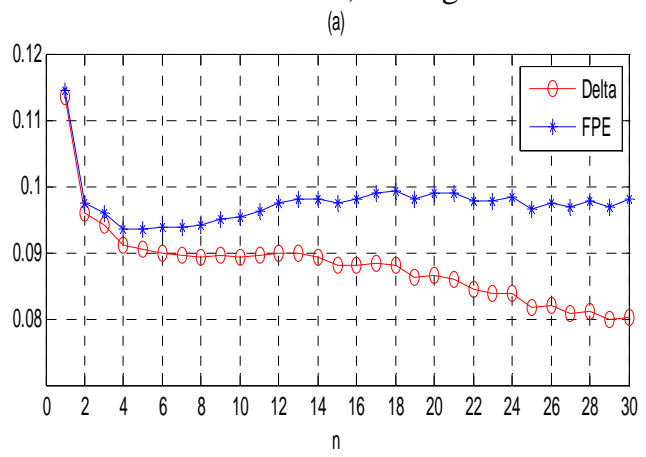

(b)

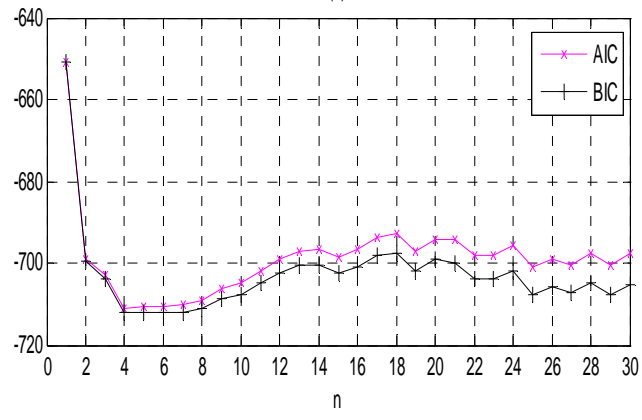

Figure.5 The order of AR model determination

From (a) and (b) in Figure.5 , it can be seen with the increasing of model order, the overall trend of $\sigma_{a}^{2}$ is downward, and when $n$ is smaller, it declined soon, with $n$ rising, the downtrend became slow. The value of criterion function decline fast when $n$ is relatively small, and soon after, it changed smoothly. However, no matter which kinds of criterion function, when $n=4$, the function value and $\sigma_{a}^{2}$ are all minimum, so $A R(4)$ is appropriate, and the model

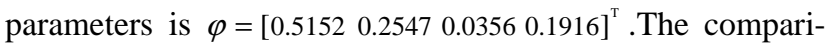
son figure between predication and original data is as Figure,6.

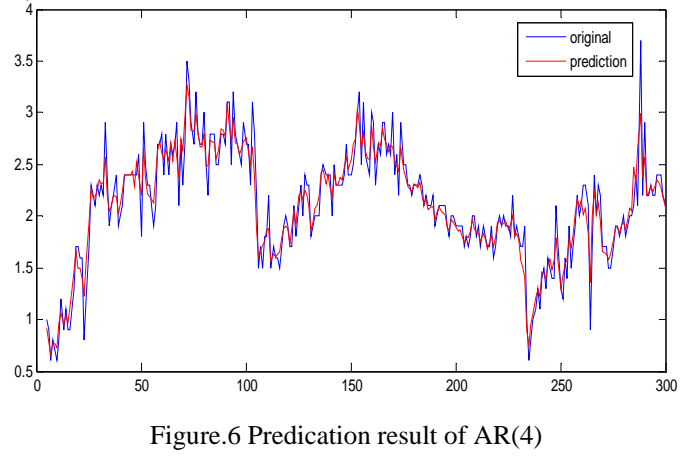

Chose the standard deviation of the prediction error as the evaluation index, the prediction error of $A R(4)$ is 0.1543 .

\section{B. Predication based on Multi-resolution analysis}

The time series of gas emission is firstly decomposed by wavelet multi-resolution analysis, which chose the wavelet function $d b 3$, and decomposed 4 layers. The decomposition result is as following.

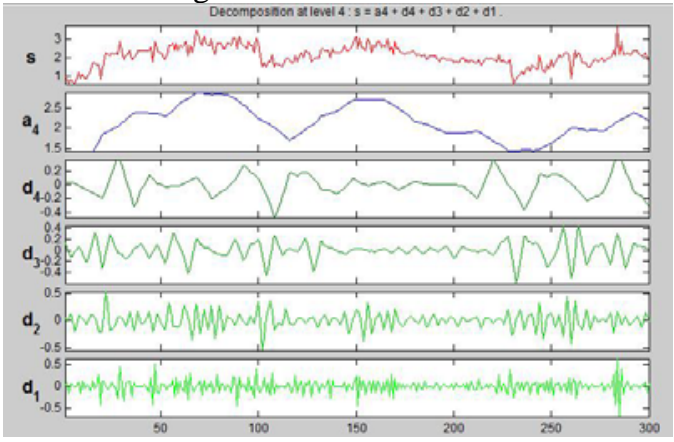

Figure.7 The decomposition diagram of multi-resolution analysis

Then the result of single branch reconstruction of each layer is predicted with AR model. synthesized the prediction results of every layers. The final comparison between forecasting result and original data is shown below.

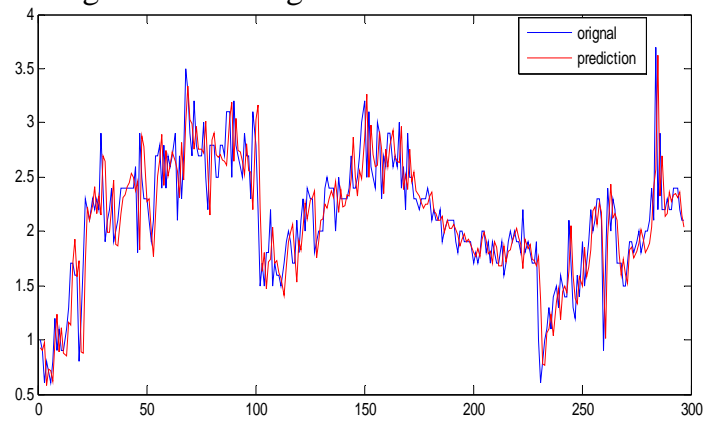

Figure.8 Prediction result based on multi-resolution analysis

The error of Prediction based on multi-resolution analysis is 0.037. Different wavelet functions and decomposition layers, predicted results are different. Through Matlab simulation and constantly testing, it can be achieved that selecting $d b 3$ and sym7, the results is better. Table I is the comparison of different wavelet function and different decomposition layers which is from 1 to 10 .

As can be seen from Table I, the predication error based on multi-resolution analysis is less than directly using $A R$ model forecasting. When the wavelet function is $d b 3$, and the decomposition layer is 4 , the error reached minimum 0.037 , meanwhile when the number of layer between $4 \sim 8$, the error value is small and stabilized.

TABLE I . The comparison of predicted results

\begin{tabular}{|c|c|c|c|c|c|}
\hline $\begin{array}{c}\text { Decomposition } \\
\text { layers }\end{array}$ & 1 & 2 & 3 & 4 & 5 \\
\hline $\mathrm{db3}$ & 0.064 & 0.057 & 0.048 & 0.037 & 0.037 \\
\hline
\end{tabular}




\begin{tabular}{|c|c|c|c|c|c|}
\hline sym7 & 0.081 & 0.049 & 0.041 & 0.040 & 0.040 \\
\hline $\begin{array}{c}\text { Decomposition } \\
\text { layers }\end{array}$ & 6 & 7 & 8 & 9 & 10 \\
\hline $\mathrm{db} 3$ & 0.037 & 0.037 & 0.037 & 0.037 & 0.037 \\
\hline sym7 & 0.040 & 0.040 & 0.040 & 0.175 & 0.243 \\
\hline
\end{tabular}

VI. CONCLUSIONS

In this paper, multi-resolution analysis was applied into the predication of gas emission, and this method was compared with $A R$ forecasting. Through the simulation, the following conclusion can be drawn. The method of predicating based on multi-resolution analysis can be used on gas emission and the accuracy is much higher than direct applying AR model. Meanwhile, the contrasts with different wavelet function and decomposition layers illustrate that the best number of layer is $4 \sim 8$.

\section{REFERENCES}

[1] K. Wang and Q.X. Yu. Non-linear Characteristics and Prediction Model of Coal and Gas Outburst. Xuzhou: China University of Mining and Technology Press, 2005
[2] H.S. Yin. Gas Time Series Analytical Method and Its Early-warning Application in Coalmine. Xuzhou: China University of Mining and Technology Press, 2010

[3] Mallat.S. “A Theory for Multi-resolution Signal Decom-position: The Wavelet Representation”. IEEE Trans on PAMI, 1989,11(7):674-693.

[4] T. Liu, X.L. Zeng, and J. Zeng. Practical Wavelet Analysis Introduction. Beijing: Defense Industry Press, 2006

[5] W. Zhou. Wavelet Analysis Applications Based on Matlab. Xi'an: Publishing House of Xi'an University of Electronic Science and Technology,2010

[6] S.Z. Yang, Y. Wu, J.P. Xuan. Time Series Analysis in Engineering Application. Wuhan: Huazhong University of Science and Technology Press, 2007

[7] G.Q. Chen, J.W. Zhao, J.J. Huang and W.L. Liu. "Matlab-based Parameter Estimation of AR Mode”. Tool Engineering. vol.39, pp.3940,2005.

[8] Daubechies.I. Ten Lectures on Wavelet. PA: Society for Industry and Applied Mathematics 1992.

[9] C. Zhang, S.B. He and H. Fan. "Linear predication based on wavelet multi-resolution analysis”. Applied Science and Technology. vol.31, pp.1415,2004

[10]K. Xu, J.W. Xu, X.J. Ban. "Forecasting of Some Non-stationary Time Series Based on Wavelet Decom-position”, Acta Electronica Sinica. vol.29, pp.566-568, 2001. 